Giulia Polo, Daniela Gueraldi, Antonella Giuliani, Laura Rubert, Chiara Cazzorla, Leonardo Salviati, Antonio Marzollo, Alessandra Biffi, Alessandro P. Burlina and Alberto B. Burlina*

\title{
The combined use of enzyme activity and metabolite assays as a strategy for newborn screening of mucopolysaccharidosis type I
}

https://doi.org/10.1515/cclm-2020-0064

Received January 17, 2020; accepted April 15, 2020

\section{Abstract}

Objectives: Mucopolysaccharidosis type I (MPS I) was added to our expanded screening panel in 2015. Since then, 127,869 newborns were screened by measuring $\alpha$-Liduronidase (IDUA) enzyme activity with liquid chromatography tandem mass spectrometry (LC-MS/MS). High false positives due to frequent pseudodeficiency alleles prompted us to develop a second-tier test to quantify glycosaminoglycan (GAG) levels in dried blood spot (DBS).

Methods: Heparan-sulfate (HS) and dermatan-sulfate (DS) were measured with LC-MS/MS after methanolysis. DBSs were incubated with methanolic- $\mathrm{HCl} 3 \mathrm{~N}$ at $65{ }^{\circ} \mathrm{C}$ for $45 \mathrm{~min}$. Chromatographic separation used an amide column with a gradient of acetonitrile and water with $10 \mathrm{mM}$ ammonium acetate in a 9-min run. The method was validated for specificity, linearity, lower limit of quantification (LOQ), accuracy and precision.

Results: Intra- and inter-day coefficients of variation were $<15 \%$ for both metabolites. Reference values in 40 healthy newborns were: HS mean $1.0 \mathrm{mg} / \mathrm{L}, 0-3.2$; DS mean $1.5 \mathrm{mg} / \mathrm{L}, 0.5-2.7)$. The two confirmed newborn MPS I

\footnotetext{
*Corresponding author: Alberto B. Burlina, Division of Inherited Metabolic Diseases, Regional Center for Expanded Neonatal Screening Department of Women and Children's Health, University Hospital of Padova, Via Orus 2/B, 35129 Padova, Italy,

E-mail: alberto.burlina@unipd.it

Giulia Polo, Daniela Gueraldi, Antonella Giuliani, Laura Rubert and Chiara Cazzorla: Division of Inherited Metabolic Diseases, Regional Center for Expanded Neonatal Screening Department of Women and Children's Health, University Hospital of Padova, Via Orus 2/B, 35129 Padova, Italy. https://orcid.org/0000-0003-3157-2979 (G. Polo)

Leonardo Salviati: Clinical Genetics Unit, Department of Women's and Children's Health, University of Padova, Padova, Italy Antonio Marzollo and Alessandra Biffi: Pediatric HematologyOncology Unit, Department of Women's and Children's Health, University of Padova, Padova, Italy

Alessandro P. Burlina: Neurological Unit, St. Bassiano Hospital, Bassano del Grappa, Italy
}

patients had elevated HS (4.9-10.4 mg/L, n.v. <3.2) and DS $(7.4-8.8 \mathrm{mg} / \mathrm{L}$, n.v. <2.7). Since its introduction in February 2019, the second-tier test reduced the recall rate from $0.046 \%$ to $0.006 \%$. Among 127,869 specimens screened, the incidence was 1:63,935 live births. Both patients started enzyme replacement therapy (ERT) within 15 days of birth and one of them received allogenic hematopoietic stem cell transplantation (HSCT) at ht age of 6 months. Conclusions: GAGs in DBS increased the specificity of newborn screening for MPS I by reducing false-positives due to heterozygosity or pseudodeficiency. Early diagnosis and therapeutical approach has improved the outcome of our patients with MPS I.

Keywords: dermatan sulfate; expanded newborn screening; glycosaminoglycans; heparan sulfate; LC-MS/MS; lysosomal disorders; lysosomal disorders newborn screening; mucopolysaccharidosis type I; second-tier test; tandem mass spectrometry.

\section{Introduction}

Mucopolysaccharidoses (MPSs) are a group of rare diseases (lysosomal storage disorders; LSDs) caused by a reduction or lack of the lysosomal enzymes that catalyze the stepwise degradation of glycosaminoglycans (GAGs) [1].

Mucopolysaccharidosis type I (MPS I) involves a deficiency of the lysosomal enzyme $\alpha$-L-iduronidase (IDUA) resulting in progressive lysosomal accumulation of dermatan and heparan sulfates with clinical manifestations characteristic of the MPS phenotype [2]. Major clinical findings include coarse facial features, skeletal and joint sequelae, a marked disproportionate short stature with short trunk and normal limbs, hepatosplenomegaly and visual impairment secondary to corneal clouding, astigmatism and/or retinopathy. Although MPS I has traditionally been divided into three broad groups based on symptom severity: Hurler (H), Hurler-Scheie (H/S) and Scheie (S) syndromes, it is increasingly considered to have a continuous disease spectrum. Hurler and Scheie 
syndromes represent phenotypes at the severe and mild extremes of the clinical spectrum, respectively, whereas the Hurler-Scheie syndrome has an intermediate phenotype. Therapeutic approaches for MPS I include enzyme replacement therapy (ERT) with L-iduronidase, allogeneic hematopoietic stem cell transplantation (HSCT) and recently, ex vivo gene therapy in autologous hematopoietic stem cells [3, 4]. The progressive nature of MPS I means that treatment success depends on early initiation. Recently, it has been demonstrated that newborn screening (NBS) for MPS I allows timely initiation of therapy, potentially changing its natural history [5].

Several analytical strategies are available for efficient full population screenings [6-10].

In Taiwan, a pilot NBS program for MPS I conducted from October 2008 to April 2013 screened 35,285 newborns and identified two patients with confirmed MPS I, estimated incidence 1:17,643 [11]. NBS programs that include MPS I have been described in the USA. In 2013, Missouri was the first state to introduce MPS I and other LSDs in a pilot screening program using a multiplexed fluorometric enzymatic assay (digital microfluidic platform) [12]. In the first 6 months, 43,701 newborns were screened; 32 were investigated for MPS I (recall rate $0.037 \%$ ). Of these, three were confirmed to have an MPS I genotype (estimated incidence 1:14,567). Of the remaining 29 patients, seven had pseudodeficiency alleles, two were carriers, 16 were falsepositives and four had results pending. In Washington State, the NBS Laboratory conducted two pilot studies on anonymous DBS using multiplex-tandem mass spectrometry (MS/MS) enzymatic activity assays that included IDUA $[7,10]$. The analytical ranges for lysosomal enzymes were 5- to 15-fold higher with MS/MS compared to the fluorimetric assays using 4-methylumbelliferyl substrates. The rate of screen positive detection was consistently lower for the MS/MS assay compared to the fluorimetric assay using a digital microfluidics platform. In 2017, a screening project conducted in Illinois on Pompe, MPS I, Gaucher, Fabry, and Niemann-Pick diseases identified one confirmed MPS I case estimated incidence 1:219,793 [13]. $\alpha$-L-iduronidase pseudodeficiencies were detected more often than true deficiencies. A recent North Carolina study employing a 2-tier method for MPS I screening in 62,734 newborns confirmed the need for second-tier testing to reduce the burden of false positives [14]. In early 2016, following systematic evidence-based studies, MPS I neonatal screening was approved by the US Secretary of the Department of Health and Human Services, and added to their Recommended Universal Screening Panel [15].

In Northeastern Italy, an NBS program for detecting LSDs including MPS I using MS/MS began in 2015.
It revealed a high frequency of IDUA pseudodeficiency alleles (benign variants) with reduced in vitro IDUA enzyme activity that resulted in a high recall rate for retesting by leucocyte enzyme activity and 24-h urinary GAGs [16, 17]. We found a high incidence of pseudodeficiency alleles (e.g. encoding p.Ala79Thr, p.His82Gln, p.Asp223Asn) in newborns of African origin, confirming previous reports [12, 13, 18].

Here, we report our experience with MPS I screening in over 125,000 newborns, initially conducted with IDUA enzyme activity alone, and subsequently with the introduction of a second-tier test to measure GAGs in DBS to reduce the false positive rate. We also report the clinical outcome of two affected patients diagnosed early through this NBS program.

\section{Materials and methods}

\section{Screening population}

Since September 2015, 127,869 consecutive newborn DBS samples were collected at the Regional Center for Expanded Newborn Screening, University Hospital of Padua. Informed consent was obtained from a parent. This center runs an expanded NBS program for infants born in northeastern Italy, where there are approximately 35,000 live births per year. Samples were collected $48 \mathrm{~h}$ after birth on the same cards used for other NBS tests $\left(903^{\mathrm{TM}}\right.$ sample collection card, GE Healthcare, Cardiff, UK); a second sample at 15 days of life was required for premature infants ( $<34$ gestational weeks and/or weight $<2000 \mathrm{~g}$ ) and after treatment in newborns with health problems (i.e. requiring transfusions or parenteral nutrition).

\section{IDUA screening assay}

IDUA enzyme activities were determined from a single DBS by stable isotope dilution flow injection analysis MS/MS (FIA-MS/MS) using a NeoLSD kit (PerkinElmer, Turku, Finland). Flow injection analyses were performed using an Acquity TQD and a Xevo TQ MS (Waters Corp., Milford, MA, USA). Enzyme activities are expressed as $\mu \mathrm{mol} /$ $\mathrm{L} / \mathrm{h}(\mu \mathrm{M} / \mathrm{h})[16]$. The mean IDUA activity was $10.12 \pm 3.47 \mu \mathrm{M} / \mathrm{h}$ and median activity was $9.67 \mu \mathrm{M} / \mathrm{h}$. Supplementary Figure S2 shows the enzyme activity distribution and scatter plot as a function of assay date over a period of 2 years.

\section{IDUA assay cut-off and the MPS I screening algorithm}

We used the cut-off values for IDUA that we had established previously and validated with known clinical case controls [16]. Based on the enzyme activity, values below $20 \%$ of the median (i.e. 0.2 multiples of the median, or $0.2 \mathrm{MOM}$ ) were considered high risk values. The cut-off value of 0.2 MOM enables detection of positive cases while 
avoiding excessive recalls. The cut-off was recalculated monthly to adjust for slight seasonal variations, which ranged from $2.3 \mu \mathrm{M} / \mathrm{h}$ in winter to $1.8 \mu \mathrm{M} / \mathrm{h}$ in summer (Supplementary Figure S2).

In the initial screening algorithm used between September 2015 and January 2019, DBS samples with IDUA enzyme activity below 0.2 MOM were retested in duplicate. If the mean retest value was below $0.2 \mathrm{MOM}$, a second spot was requested. If the activity of the second spot was also below the cut-off, the infant was referred to our unit for confirmatory testing and clinical follow-up.

In February 2019, the screening algorithm was modified to include a second-tier test to measure GAG concentrations in the DBS samples that have IDUA activity below 0.2 MOM. In the new algorithm, only patients with low IDUA activity and elevated DBS GAGs are referred for confirmatory testing and clinical follow-up (Supplementary Figure S5).

\section{Follow-up and confirmatory tests}

Confirmatory testing for newborns with positive screening results included urinary GAGs (heparan [HS] and dermatan sulfate [DS] determined using an LC-MS/MS method based on that described by Auray-Blais et al. [19], with modifications (see Supplementary material) and targeted gene sequencing.

\section{Second-tier testing by measuring GAGs in DBSs}

Samples: We measured DBS GAGs in residual neonatal DBS specimens with IDUA activity below the referral cut-off and confirmed them by mutation analysis. Reference values were established in DBS from healthy anonymous neonates $(n=40)$ with normal IDUA activity.

Chemicals and stock solution preparation: DS, HS and chondroitin sulfate A (CS) were purchased from Sigma Aldrich (Saint-Louis, MO, USA); LC-MS grade ACN and ammonium acetate were from Merck Millipore. Methanolic hydrochloric acid ( $\mathrm{HCl}) 3$ N, methanol-d4 (99.8 atom\% D) and acetyl chloride were also from Sigma Aldrich. Stock and intermediate solutions of DS, HS and CS were prepared gravimetrically in water at $10 \mathrm{mg} / \mathrm{mL}$ and $1 \mathrm{mg} / \mathrm{mL}$, respectively, and stored at $-20{ }^{\circ} \mathrm{C}$. Deuterated internal standards were prepared in-house by deuterio-methanolysis of DS, CS and HS standards as previously described by Zhang et al. [19, 20]. Stock solutions were prepared in water at $600 \mathrm{mg} / \mathrm{L}$ and stored at $-20^{\circ} \mathrm{C}$.

Standard curve and quality controls: A seven-level calibration curve was prepared with a mixture of DS and HS standards added to pooled human whole blood to obtain the concentrations: $0,1.56,3.13$, 6.25, 12.5, 25 and $50 \mathrm{mg} / \mathrm{L}$ for HS and DS; a separate calibration curve was prepared for CS by adding the standard to obtain the concentrations: $0,3.13,6.25,12.5,25,50$ and $100 \mathrm{mg} / \mathrm{L}$. Two quality controls containing DS and HS were prepared from a mixture of working solutions: medium concentration, $10 \mathrm{mg} / \mathrm{L}$ of each; high concentration, $40 \mathrm{mg} / \mathrm{L}$ of each). Fifty microliters $(50 \mu \mathrm{L})$ of spiked blood were then spotted onto a sample collection card, dried at room temperature and stored at $-20^{\circ} \mathrm{C}$ until analysis.
Sample preparation and analysis: Two 3.2-mm punches from each DBS were deposited in one well of a 96-well plate and $200 \mu \mathrm{L}$ of methanolic $\mathrm{HCl} 3 \mathrm{~N}$ solution added. The plate was incubated at $65{ }^{\circ} \mathrm{C}$ for $45 \mathrm{~min}$ with intermittent orbital shaking at $400 \mathrm{rpm}(30 \mathrm{~s}$ every $10 \mathrm{~min}$ ), after which all the supernatant was transferred to a new plate and evaporated under a stream of nitrogen; samples were resuspended in $100 \mu \mathrm{L}$ of a solution containing deuterated internal standard (DS ${ }^{2} \mathrm{H}_{6}$ and $\mathrm{HS}^{2} \mathrm{H}_{6}$ ) at $1 \mathrm{mg} / \mathrm{L}$ in $\mathrm{ACN}: \mathrm{H}_{2} \mathrm{O}(90: 10 \mathrm{v} / \mathrm{v})$ with $10 \mathrm{mM}$ ammonium acetate solution.

Simultaneous analysis of DS, CS, HS disaccharides and deuterated internal standards was performed on a Xevo TQ-S micro MS/MS combined to an Acquity I-Class UPLC system (Waters Corp.). Mass spectrometry acquisition parameters and liquid chromatography parameters are presented in Table 1.

Method validation: Within-run and between-run precision was determined by preparing and analyzing each QC sample in ten replicates on the same batch and in single for 10 consecutive working days. Method accuracy was assessed by recovery studies on the QC samples. Accuracy was expressed as percent relative error (\%RE), calculated by subtracting the nominal value from the mean observed value, dividing by the nominal value and then multiplying by 100: ([mean-nominal]/[nominal] $\times 100)$. The linearity of the seven-point calibration curve was evaluated. The stability of HS and DS in DBS specimens stored at different temperatures (ambient, refrigerated $\left(2-8{ }^{\circ} \mathrm{C}\right),-20{ }^{\circ} \mathrm{C}$ and $\left.-80{ }^{\circ} \mathrm{C}\right)$ was evaluated by periodically sampling and analyzing three DBS specimens stored under each condition for up to 6 months. QC DBSs were used to assayed stability. The limit of detection (LOD) and limit of quantification (LOQ) were defined as three and 10 times the residual standard deviation $(\sigma)$ of $y$-intercepts of regression lines divided by the slope (S).

Data analysis: Analyte peak areas were measured with TargetLynx 4.1 software (Waters, USA) and normalized to the internal standard peak area. Selected disaccharides were quantified using the calibration curves described previously. Quantification was performed on a seven-point calibration curve prepared gravimetrically comparing the signals of the GAG-specific dimer products with those of the internal standards (Analyte Area/IS Area $=$ Response), and conversion to concentration by response of calibration curves. The calibration curve was linear with a $1 / x$ weighing function, and the origin was included. The endogenous level of each GAG was calculated from the y-intercept concentrations for multiple calibration curves $(n=3)$. Statistical analysis was performed using GraphPad Prism software (GraphPad Software Inc., San Diego, CA, USA).

\section{Results}

\section{Second-tier test by DBS GAGs analysis}

To improve the performance of NBS for MPS I and reduce the large number of recalls, we recently developed a second-tier test based on quantification of GAG levels in DBSs by methanolysis followed by LC-MS/MS analysis. 
Table 1: $L C$ and MS/MS parameters used for GAGs analysis.

\begin{tabular}{|c|c|c|c|c|c|c|c|c|}
\hline \multicolumn{3}{|l|}{ HPLC parameters } & \multicolumn{6}{|c|}{ MS/MS parameters } \\
\hline Column & \multicolumn{2}{|c|}{$\begin{array}{l}\text { Acquity UPLC BEH } \\
\text { AMIDE } 2.1 \times 150 \mathrm{~mm}\end{array}$} & \multicolumn{2}{|l|}{ Mass spectrometer } & \multicolumn{4}{|c|}{ Waters Xevo TQ-S MICRO } \\
\hline $\begin{array}{l}\text { Column } \\
\text { temperature }\end{array}$ & \multicolumn{2}{|l|}{$30^{\circ} \mathrm{C}$} & \multicolumn{2}{|l|}{ Ionization mode } & \multicolumn{4}{|l|}{$\mathrm{ESI}+$} \\
\hline Mobile phase A & $\begin{array}{l}90: 10 \\
\mathrm{H}_{2} \mathrm{O}: \mathrm{ACN}+10 \mathrm{mM} \\
\mathrm{CH}_{3} \mathrm{COONH}_{4}\end{array}$ & & \multicolumn{2}{|l|}{ Captiary voliase } & \multicolumn{2}{|l|}{$3.30 \mathrm{KV}$} & & \\
\hline Mobile phase B & \multicolumn{2}{|l|}{$\begin{array}{l}\mathrm{ACN}: \mathrm{H}_{2} \mathrm{O}+10 \mathrm{mM} \\
\mathrm{CH}_{3} \mathrm{COONH}_{4}\end{array}$} & & & & & & \\
\hline \multirow[t]{5}{*}{ Gradient } & $\underline{\text { Time }}$ & $\%$ B & \multicolumn{2}{|c|}{ Source temperature } & \multicolumn{2}{|l|}{$150^{\circ} \mathrm{C}$} & & \\
\hline & Initial-1.50 min & 100 & \multicolumn{2}{|c|}{ Desolvation temperature } & \multicolumn{2}{|l|}{$650^{\circ} \mathrm{C}$} & & \\
\hline & $1.50-4.00 \mathrm{~min}$ & 90 & \multicolumn{2}{|l|}{ Cone gas flow } & \multicolumn{2}{|l|}{$50 \mathrm{~L} / \mathrm{h}$} & & \\
\hline & $4.00-7.50 \mathrm{~min}$ & 60 & \multicolumn{2}{|l|}{ Dwell time } & \multicolumn{2}{|l|}{$0.042(\mathrm{~s})$} & & \\
\hline & $7.50-9.00 \mathrm{~min}$ & 100 & \multicolumn{2}{|c|}{ Desolvation gas flow } & \multicolumn{2}{|l|}{$1000 \mathrm{~L} / \mathrm{h}$} & & \\
\hline Flow rate & \multicolumn{2}{|l|}{$0.5 \mathrm{~mL} / \mathrm{min}$} & Compound & Parent $(m / z)$ & $\begin{array}{l}\text { Daughter } \\
(\mathrm{m} / \mathrm{z})\end{array}$ & Cone (V) & Collision (V) & $\begin{array}{l}\text { Retention } \\
\text { time (min) }\end{array}$ \\
\hline $\begin{array}{l}\text { Injection } \\
\text { volume }\end{array}$ & \multicolumn{2}{|l|}{$\begin{array}{l}1 \mu \mathrm{L} \text { urine } / 5 \mu \mathrm{L} \\
\text { DBS }\end{array}$} & DS_CS & $426.1>$ & 236.1 & 30 & 8 & $3.55 \_4.44$ \\
\hline Run time & \multicolumn{2}{|l|}{$9 \min$} & $\mathrm{DS}^{2} \mathrm{H}_{6-} \mathrm{CS}^{2} \mathrm{H}_{6} \mathrm{IS}$ & $432.3>$ & 239.2 & 30 & 9 & $3.55 \_4.44$ \\
\hline \multirow{2}{*}{$\begin{array}{l}\text { Autosampler } \\
\text { temperature }\end{array}$} & \multirow{2}{*}{\multicolumn{2}{|c|}{$10^{\circ} \mathrm{C}$}} & HS & $384.1>$ & 162.1 & 25 & 14 & 6.07 \\
\hline & & & $\mathrm{HS}^{2} \mathrm{H}_{6}$ IS & $390.2>$ & 162 & 40 & 14 & 6.07 \\
\hline
\end{tabular}

\section{Optimization of the methanolysis reaction}

The reaction conditions for GAGs methanolysis were optimized at $45 \mathrm{~min}$ at $65{ }^{\circ} \mathrm{C}$ to maximize the concentrations of DS and HS. Mass spectra from methanolysates of the GAG standards shown predominantly the protonated form for all the standards; sodiated molecular ions were not detected. Figure 1 shows the chromatographic separation of disaccharides from DS, HS, CS, and the respective internal standards performed on a BEH-Amide column over a run time of $9 \mathrm{~min}$, including the re-equilibration step.

\section{Method validation}

Results of method validation studies conducted on DBS are summarized in Table 2. Bias and precision were evaluated by analyzing QC samples at low and high concentrations. Evaluation of within-day and between-day precision demonstrated CVs $<15 \%$ at two concentration levels. LODs for DS, HS, and CS disaccharides were $0.1,0.2$ and $0.2 \mathrm{mg} / \mathrm{L}$, respectively, whereas LLOQs were $0.4,0.5$ and $0.7 \mathrm{mg} / \mathrm{L}$. Good linearity was obtained in the range of investigated concentrations for the three standards (HS, $\mathrm{r}^{2}=0.998$; DS, $\left.r^{2}=0.998 ; C S, r^{2}=0.992\right)$ (Supplementary Figure S4). DBS specimens were useful for HS and DS determination for at least 6 months of storage under ambient conditions, refrigerated, frozen $\left(-20^{\circ} \mathrm{C}\right)$ or frozen $\left(-80^{\circ} \mathrm{C}\right)$. Specimens were also subjected to up to six freeze/thaw cycles.

Reference ranges were determined in 40 healthy newborns (HS mean $1 \mathrm{mg} / \mathrm{L}$, range 0-3.2; DS mean $1.5 \mathrm{mg} / \mathrm{L}$, range 0.5-2.7).

We measured GAGs in DBS from 22 previously identified newborns with low IDUA enzyme activity and known genomic status. Two of the patients had two pathological alleles (true positives), 17 had pseudodeficiency, one was a carrier and two carried variants of unknown significance). Only the two neonates with two pathogenic alleles had high levels of HS (4.9-10.4 mg/L) and DS (7.4$8.8 \mathrm{mg} / \mathrm{L}$ ); all other samples had GAGs within the normal range (mean HS $1.7 \mathrm{mg} / \mathrm{L}$, range 1.1-3.1; mean DS $1.9 \mathrm{mg} / \mathrm{L}$, range 1.5-2.9) (Figure 2).

\section{Potential interference}

Potential interference due to the presence of heparin anticoagulant in venous blood draws was evaluated by preparing DBS specimens from venous blood collected with lithium heparin (Hep DBS) versus blood collected via a 


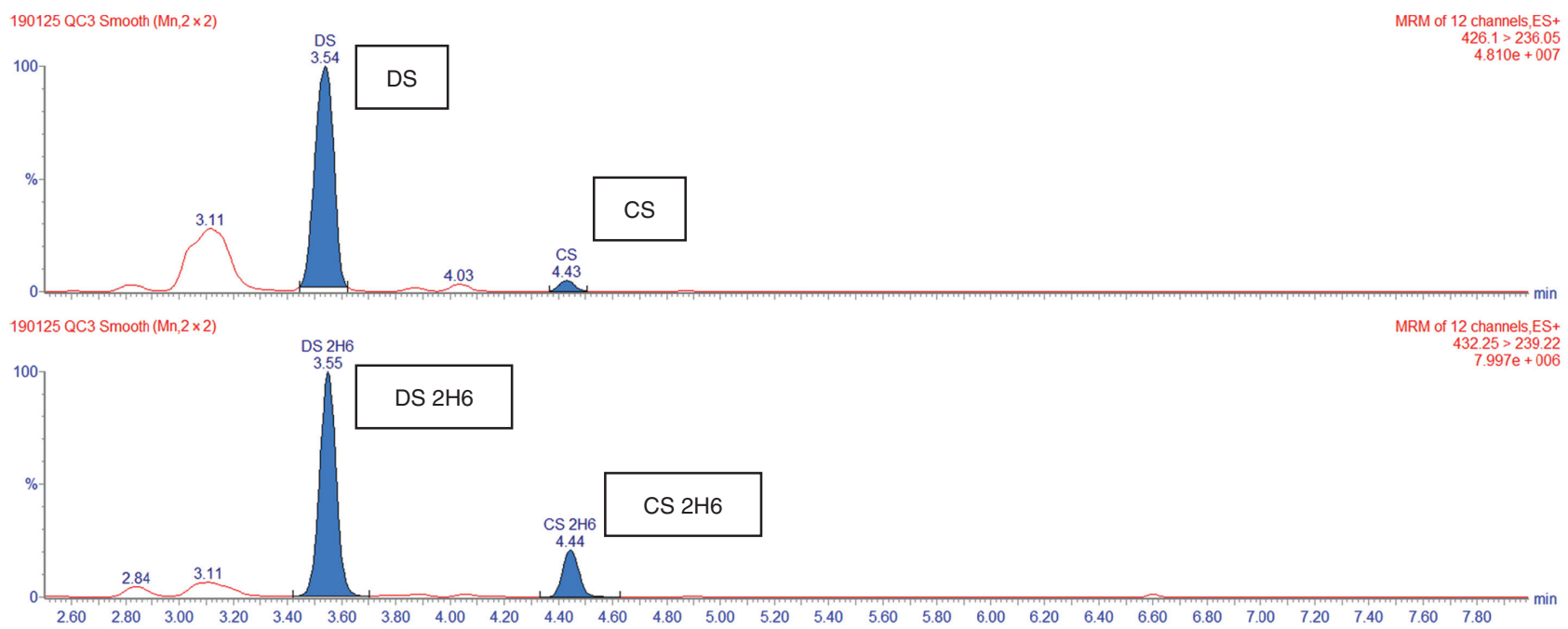

190125 QC3 Smooth (Mn,2×2)

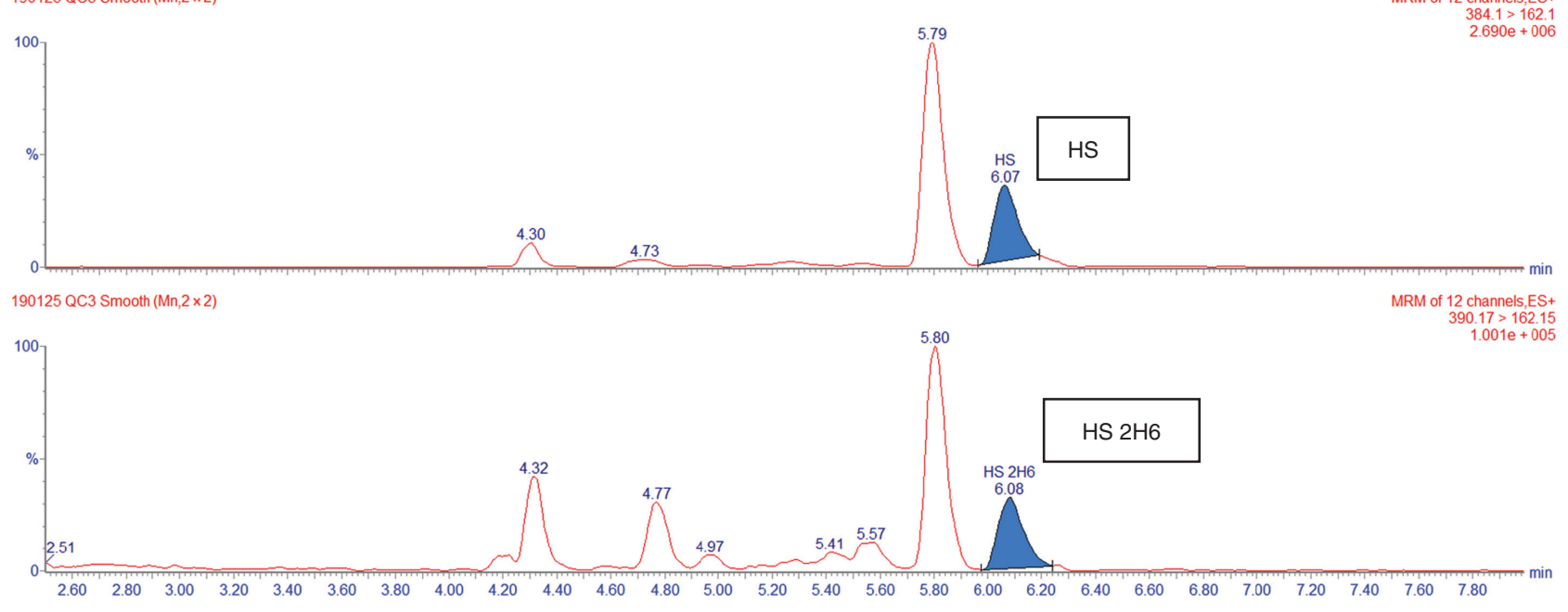

Figure 1: Chromatographic separation of GAG disaccharides and internal standards in a high QC DBS on the BEH Amide column over a 9-min run.

MRM acquisition window 2.5-8 min. DS, dermatan sulfate; $\mathrm{CS}$, chondroitin sulfate; $\mathrm{HS}$, heparan sulfate; $\mathrm{DS}^{2} \mathrm{H}_{6}$, DS internal standard; $\mathrm{CS}^{2} \mathrm{H}_{6}$, CS internal standard IS; $\mathrm{HS}^{2} \mathrm{H}_{6}$, HS internal standard; MRM, multiple reaction monitoring.

Table 2: Analytical performance of the LC-MS/MS method: intra- and inter-assay precision (percent coefficient of variation, CV \%) at three concentration levels and recovery $\%$.

\begin{tabular}{|c|c|c|c|c|c|c|c|c|}
\hline & \multicolumn{2}{|c|}{ QC endogenous } & \multicolumn{3}{|c|}{ Medium QC (endogenous $+10 \mathrm{mg} / \mathrm{L}$ ) } & \multicolumn{3}{|c|}{ High QC3 (endogenous $+40 \mathrm{mg} / \mathrm{L}$ ) } \\
\hline & $\begin{array}{r}\text { Mean } \pm \mathrm{SD} \\
\mathrm{mg} / \mathrm{L}\end{array}$ & $\begin{array}{r}\text { Precision } \\
\text { CV, } \%\end{array}$ & $\begin{array}{r}\text { Mean } \pm \mathrm{SD} \\
\mathrm{mg} / \mathrm{L}\end{array}$ & $\begin{array}{r}\text { Precision } \\
\text { CV, \% }\end{array}$ & $\begin{array}{r}\text { Recovery, } \\
\%\end{array}$ & $\begin{array}{r}\text { Mean } \pm \text { SD } \\
\mathrm{mg} / \mathrm{L}\end{array}$ & $\begin{array}{r}\text { Precision } \\
\text { CV, \% }\end{array}$ & $\begin{array}{r}\text { Recovery, } \\
\%\end{array}$ \\
\hline \multicolumn{9}{|c|}{ Intra-assay, $(n=11)$} \\
\hline DS & $1.60 \pm 0.13$ & 8.1 & $10.9 \pm 1.2$ & 11 & 93 & $42.9 \pm 2.9$ & 9.7 & 103.2 \\
\hline HS & $0.9 \pm 0.2$ & 22.2 & $10.1 \pm 0.95$ & 9.41 & 92 & $39.6 \pm 3.2$ & 9.8 & 96.7 \\
\hline \multicolumn{9}{|c|}{ Inter-assay, $(\mathrm{n}=7)$} \\
\hline DS & $1.80 \pm 0.29$ & 16.3 & $11.2 \pm 1.32$ & 11.9 & 93.9 & $42.9 \pm 4.9$ & 11.4 & 102.7 \\
\hline HS & $0.72 \pm 0.17$ & 23.8 & $10.4 \pm 1.30$ & 12.4 & 96.6 & $35.6 \pm 3.5$ & 9.8 & 87.3 \\
\hline
\end{tabular}

CV\%, percentage coefficient of variation; LC-MS/MS, liquid chromatography tandem mass spectrometry; SD, standard deviation. 


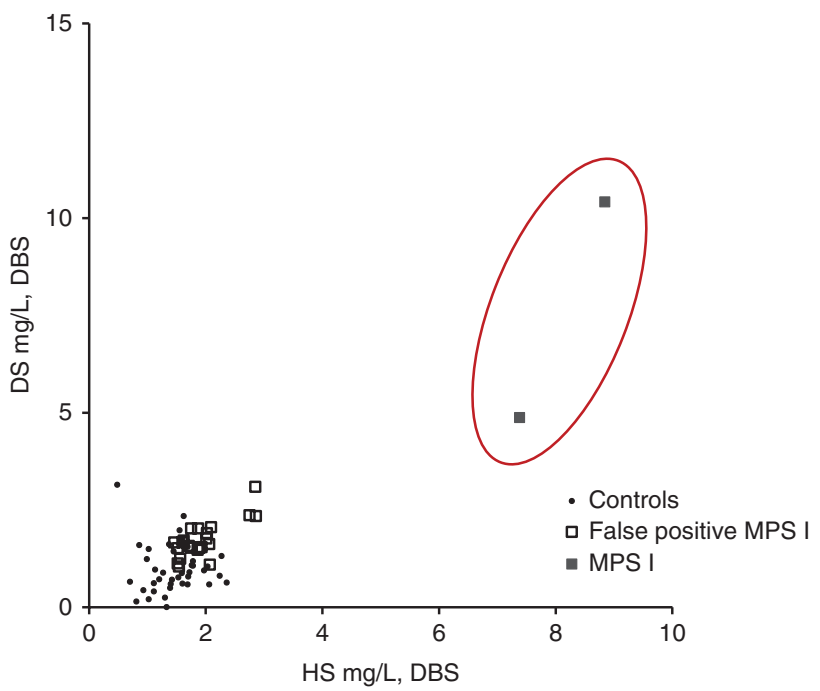

Figure 2: HS and DS concentrations in DBS of controls (black dots), false positive MPS I newborns (empty square) and newborns with confirmed MPS I (black square).

finger prick from the same donor $(n=4)$. HS was 100-fold higher in the DBS spotted from heparin blood, compare to the finger prick DBS (Supplementary Table S3). Heparin interferes with the measurement of HS because methanolysis produces a common IdoA-GlcN disaccharide that is monitored by LC-MS/MS.

\section{MPS I screening results}

\section{Before introduction of the second-tier test (September 2015 to January 2019)}

Since September 2015, a total of 127,869 neonates have been screened for MPS I in northeastern Italy; up until January 2019, 110,244 neonates were screened based on IDUA enzyme activity alone (first-tier only) and 52 putatively positive newborns were identified (mean retest values below $0.2 \mathrm{MOM}$ ) and recalled for a second DBS card (recall rate $0.046 \%$ ). Of these patients, 23/52 had very low enzyme activity (Group 1) with values below $10 \%$ of the median (mean $0.53 \mu \mathrm{mol} / \mathrm{L} / \mathrm{h}$ range $0.10-1.17 \mu \mathrm{mol} / \mathrm{L} / \mathrm{h}$ ), $29 / 52$ had marginally low activity between $10 \%$ and $20 \%$ of the median (Group 2), with mean $1.69 \mu \mathrm{mol} / \mathrm{L} / \mathrm{h}$, range 1.19-2.31 $\mu \mathrm{mol} / \mathrm{L} / \mathrm{h}$. On IDUA retesting, $26 \mathrm{had}$ confirmed low enzyme activity: 21/23 were from Group 1 (91\%) and 5/29 were from Group 2 (17\%).

All 26 positive neonates subsequently underwent full diagnostic assessment including determination of IDUA in lymphocytes, urinary GAGs by LC-MS/MS analysis and genotyping. Data are summarized in Table 3. Two newborns (2/26) had two pathogenic alleles previously reported in MPS I (IDUA-02 and IDUA-04); 21/26 patients had genotypes that were not consistent with MPS I: pseudodeficiency alleles $(n=17)$; compound heterozygotes with one pathogenic allele and one pseudodeficiency allele $(n=2)$; gene variants of unknown significance $(n=2)$; genetic analysis was not available for three out of 26 patients (Table 3). All patients with genotypes that were not consistent with MPS I also had normal results for GAGs in DBSs.

\section{After introduction of the second-tier test (Since February 2019)}

Since February 2019, the MPS I screening workflow used in northeastern Italy has included the second-tier test for DBS GAGs. During this period, we screened 17,625 newborns and identified five who had low IDUA activity but normal DBS GAGs (Table 4); therefore, no diagnostic testing was required, per protocol. One exception was made to the protocol for patient STT-P1, who had very low IDUA activity $(0.21 \mu \mathrm{mol} /$ $\mathrm{L} / \mathrm{h}$ ), despite normal DBS GAG levels. The very low IDUA activity was confirmed in lymphocytes $(0.9 \mathrm{nmol} / \mathrm{h} / \mathrm{mg}$, normal values $7.47-29.74 \mathrm{nmol} / \mathrm{h} / \mathrm{mg}$ ); however, urinary GAG levels were normal and genotype analysis revealed pseudodeficiency. Thus, the recall rate for MPS I was $0.006 \%$.

\section{Clinical status of patients identified with MPS I}

Since 2015, two confirmed cases of MPS I were identified among 127,869 specimens analyzed, incidence 1:63,935 live births. Both patients started ERT (100 U/kg/ week) within 15 days of birth. P1 (IDUA-04), a female of Moroccan descent identified in 2017 (currently 3 years old), is homozygous for the p.P533R mutation associated with the Hurler/Scheie phenotype [21]. Asymptomatic at birth, her urinary DS and HS levels normalized after 1 month on ERT. P2 (IDUA-02), a female of Italian descent identified in 2018 (currently 2 years old) is compound heterozygous for two mutations with severe phenotypes (c.46_57del12/p.Y201X). At birth, she presented with corneal clouding, mildly coarse facial features and moderate sensorineural hearing loss. ERT therapy normalized HS and DS levels after 3 months. At 6 months of age she received an allogenic HSCT. At present, both patients are asymptomatic, with normal psychomotor development and brain MRI. 


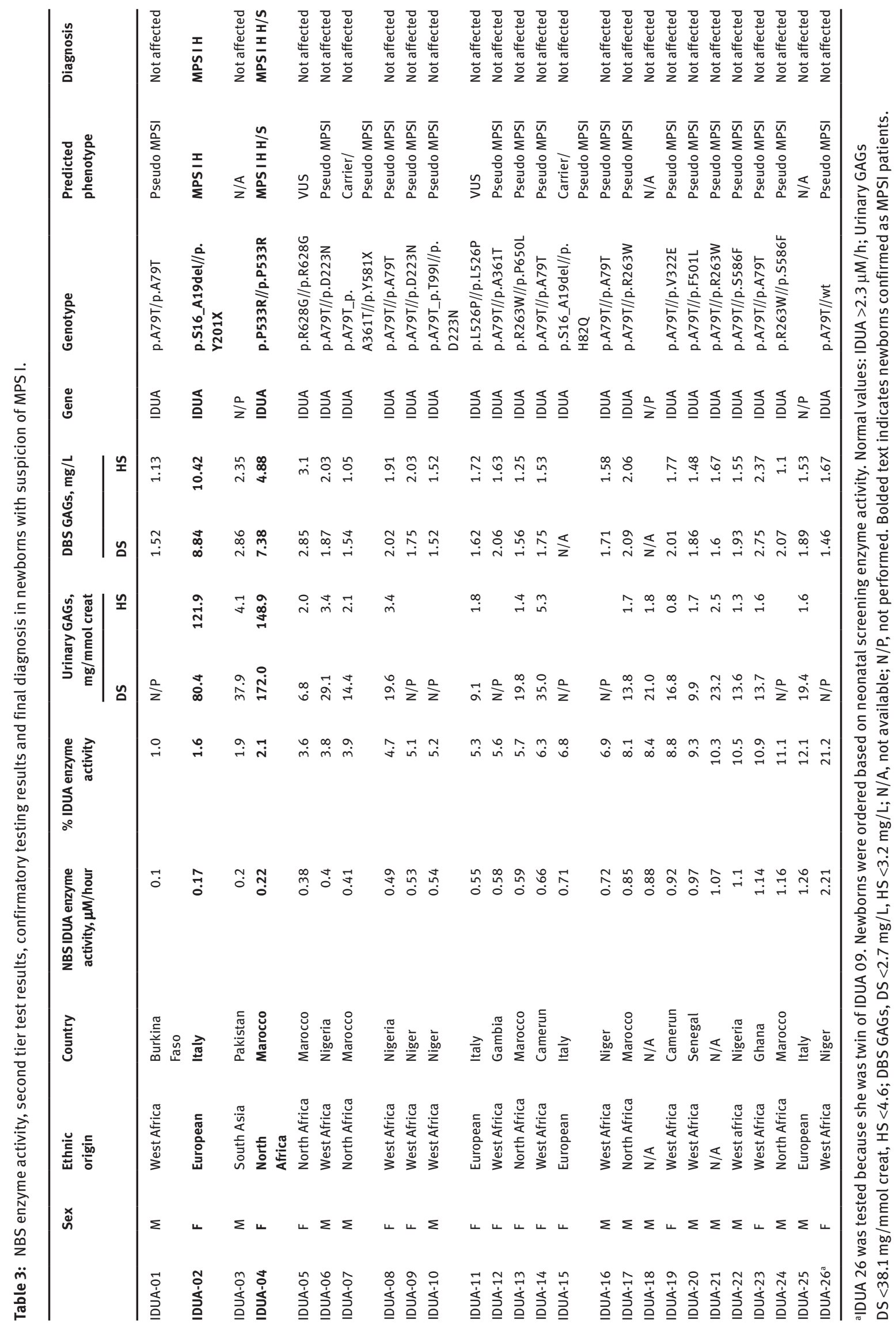


Table 4: HS and DS Second tier test results of five neonatal DBS with low IDUA activity.

\begin{tabular}{lrrr}
\hline & $\begin{array}{r}\text { DS, } \\
\mathbf{m g} / \mathrm{L}\end{array}$ & $\begin{array}{r}\text { HS, } \\
\mathbf{m g} / \mathrm{L}\end{array}$ & $\begin{array}{r}\text { IDUA, } \\
\boldsymbol{\mu M} / \text { hour }\end{array}$ \\
\hline STT-P1 & 2.06 & 1.23 & 0.215 \\
STT-P2 & 1.79 & 0.77 & 1.12 \\
STT-P3 & 3.14 & 1.04 & 1.56 \\
STT-P4 & 2.6 & 0.89 & 1.7 \\
STT-P5 & 2.49 & 1.38 & 2.05 \\
\hline
\end{tabular}

DS, dermatan-sulfate; HS, heparan-sulfate; STT, second tier test.

\section{Discussion}

The recent introduction of MS/MS methods to analyze metabolites in DBS has facilitated and expanded the number of inherited diseases detectable in newborn screening programs. Pilot screening programs for lysosomal disorders have been implemented in several countries worldwide [9].

In 2014, expanded neonatal screening for amino acid, organic acid and $\beta$-oxidation disorders was established in our laboratory in Northeastern Italy, and recently a lysosomal diseases panel has been proposed [16, 17]. Our experience shows that NBS for LSDs can be beneficial where there is an established treatment that can delay or halt progression of the disease and/or improve long-term outcomes, particularly when treatment is initiated before damage becomes irreversibly. We also underscore the need to reduce the number of false positives, which lead to unnecessary anxiety and expensive follow-up. In order to achieve this, new strategies that include second-tier tests have been employed [22, 23]. Second-tier biochemical tests that measure lysosphingolipids such as LysoGb1 in Gaucher disease, LysoGb3 in Fabry disease and psychosine in Krabbe disease clearly reduce the number of false positives in LSD newborn screening [17, 24, 25].

Our MPS I screening has identified a high number of false positives, mainly due to the presence of pseudodeficiency alleles $[13,16,18]$. The frequency of pseudodeficiency varies by population: a high incidence of was found in African and African-American populations [5, $17,18]$, conversely, no pseudodeficiency was detected in a recent study in Taiwan [26].

Strategies to reduce recall rates in MPS I screening have included the use of second-tier biochemical metabolite assays, genetic testing and post-analysis of NBS data with statistical tools. The multivariate pattern recognition software Collaborative Laboratory Integrated Reports (CLIR) is freely available and widely used for postanalytical interpretation. CLIR can improve the quality of newborn screening conducted with tandem mass spectrometry [27], and can be used as a decision tool to identify candidate patients for second-tier testing. IDUA gene sequencing has been proposed as a second-tier option. The North Carolina NBS program includes CLIR analysis and DNA sequencing in their screening algorithm $[14,28]$. They successfully demonstrate the utility of a two-tier approach to reduce the number of newborns referred for follow-up by excluding specimen that have low IDUA activity but carry only pseudodeficiency variants.

Biochemical quantification of the primary GAG storage products HS and DS in DBS has been proposed. Until recently, there was limited knowledge of the quantity of disaccharides in DBS. In 2010, Tomatsu et al. reported a method to quantify HS and DS in DBS by LC-MS/MS after enzymatic digestion [29]. This methodology allows sensitive quantification of GAGs, but involves long incubation periods and expensive reagents. In 2012, De Ruijter et al. used this method to show that GAGs were elevated in newborn DBS of patients with MPS I, MPS II and MPS III [30], suggesting that GAG determination could be a potential first-tier test for MPS newborn screening. In 2017, a similar study by Kubaski et al. confirmed the validity of measuring GAGs for identifying neonates with MPSs [31]. However, application as a first-tier test NBS is limited by the long run time and high reagent costs. Rinaldo et al. used GAG quantification as a second-tier test on specimens that had been identified as at risk for MPS I by post-analysis biostatistical analysis (CLIR) among 55,161 neonates screened in Kentucky [22]. This second-tier assay uses enzymatic digestion followed by MS. Implementing it in the workflow reduced the number of recalls to only two newborns; MPS I was confirmed in one.

Two-tier screening with sequential determination of enzyme activity and multiple GAGs has been proposed as the gold standard for the diagnosis of patients affected by mucopolysaccharidosis [32, 33]. More recently, Peck et al. used GAG quantification in DBS as a second-tier biomarker test for 1213 newborns identified as at risk of MPS I based on first-tier NBS [34]. GAGs analysis accurately discriminated between patients with confirmed MPS I and false-positive cases due to pseudodeficiency or heterozygosity, and increased the specificity of newborn screening for MPS I.

Our NBS program for MPS I revealed a high incidence of IDUA pseudodeficiency in the screened population. In February 2019, we introduced a second-tier test to measure HS and DS in DBSs by LC-MS/MS after chemical hydrolysis (methanolysis). This technique was originally developed elsewhere for use on CSF and urine [20, 35-37]; we have 
optimized it for DBS. The simple and rapid method entails direct incubation of DBS samples in methanolic- $\mathrm{HCl}$, without an extraction step. We validated the assay for clinical application by establishing the overall precision, accuracy, linearity and potential limits. Intra- and between-day reproducibility of HS and DS quantification is high. We propose this method as an economical and rapid alternative to the enzymatic digestion assay currently used.

After introducing this second-tier test into our MPS I screening algorithm, the recall rate decreased from $0.046 \%$ to $0.006 \%$. Retrospective testing of GAGs in DBS from patients known to carry single pathogenic alleles or to have pseudodeficiency alleles was able to exclude any severe form of MPS I with a negative predictive value of $100 \%$. This is consistent with reports that sequential IDUA enzyme activity and GAGs analysis reduce false positives.

This method may be applicable to second-tier testing for MPS II, MPS III and MPS VI in NBS; however, its use for MPS IV might be limited by poor quantification of KS using methanolysis [36].

This method has several potential limitations. First, the use of heparin as an anticoagulant in sample collection interferes with HS quantification because common species are generated during methanolysis [19], a phenomenon not observed with enzymatic digestion. Second, the reference ranges identified in this study may be limited to our laboratory as the purity of standard used for the calibration curves is not reported for all analytes. Moreover, biochemical analysis may differ in terms of the targeted metabolites (GAGs vs. non-reducing end oligosaccharides) and analytical methods used (GAG analysis after methanolysis vs. enzymatic digestion; LC-MS/MS vs. fluorimetric analysis). Zhang et al. compared urinary GAGs concentrations obtained with methanolysis with those with enzymatically derived dimers. They concluded that reported reference values should only be used as a guide, because inter-laboratory and inter-method differences in accuracy may affect results [19]. It is recommended that laboratoryspecific age-matched control ranges be established from a normal population. Third, newborns with attenuated MPS I may be misdiagnosed using this screening algorithm; more data must be collected on GAGs in attenuated MPS I.

In conclusion, although experience with MPS I screening is still limited, early identification and intervention has improved the outcome of our patients with MPS I. GAGs analysis in DBSs discriminates patients with confirmed MPS I and false positives, thereby increasing the specificity of MPS I newborn screening.

Acknowledgments: We thank Richard Vernell, an independent medical writer, who provided medical writing support funded by Cometa A.S.M.M.E. - Associazione Studio Malattie Metaboliche Ereditarie - ONLUS.

Author contributions: All authors have accepted responsibility for the entire content of this manuscript and approved its submission.

Research funding: This work was supported by the Cometa A.S.M.M.E. - Associazione Studio Malattie Metaboliche Ereditarie - ONLUS.

Employment or leadership: None declared.

Honorarium: None declared.

Competing interests: Authors state no conflict of interest. Informed consent: Informed consent was obtained from all individuals included in this study.

Ethical approval: Research involving human subjects complied with all relevant national regulations, institutional policies and is in accordance with the tenets of the Helsinki Declaration (as revised in 2013), and has been approved by the authors' Institutional Review Board or equivalent committee.

\section{References}

1. Muenzer J. Overview of the mucopolysaccharidoses. Rheumatology 2011;50:v4-12.

2. Muenzer J, Wraith JE, Clarke LA, International Consensus Panel on Management and Treatment of Mucopolysaccharidosis I. Mucopolysaccharidosis I: management and treatment guidelines. Pediatrics 2009;123:19-29.

3. Sawamoto K, Chen H-H, Alméciga-Díaz CJ, Mason RW, Tomatsu S. Gene therapy for mucopolysaccharidoses. Mol Genet Metab 2018;123:59-68.

4. Giugliani R, Federhen A, Vairo F, Vanzella C, Pasqualim G, da Silva $L M$, et al. Emerging drugs for the treatment of mucopolysaccharidoses. Expert Opin Emerg Drugs 2016;21:9-26.

5. Clarke LA, Atherton AM, Burton BK, Day-Salvatore DL, Kaplan P, Leslie ND, et al. Mucopolysaccharidosis type I newborn screening: best practices for diagnosis and management. J Pediatr 2017;182:363-70.

6. Orsini JJ, Martin MM, Showers AL, Bodamer OA, Zhang XK, Gelb $\mathrm{MH}$, et al. Lysosomal storage disorder $4+1$ multiplex assay for newborn screening using tandem mass spectrometry: application to a small-scale population study for five lysosomal storage disorders. Clin Chim Acta 2012;413:1270-3.

7. Scott CR, Elliott S, Buroker N, Thomas LI, Keutzer J, Glass M, et al. Identification of infants at risk for developing Fabry, Pompe, or mucopolysaccharidosis-I from newborn blood spots by tandem mass spectrometry. J Pediatr 2013;163:498-503.

8. Sista RS, Eckhardt AE, Wang T, Graham C, Rouse JL, Norton SM, et al. Digital microfluidic platform for multiplexing enzyme assays: implications for lysosomal storage disease screening in newborns. Clin Chem 2011;57:1444-51.

9. Gelb MH. Newborn screening for lysosomal storage diseases: methodologies, screen positive rates, normalization of datasets, second-tier tests, and post-analysis tools. Int J Neonatal Screen 2018;4:23. 
10. Elliott S, Buroker N, Cournoyer JJ, Potier AM, Trometer JD, Elbin $C$, et al. Pilot study of newborn screening for six lysosomal storage diseases using tandem mass spectrometry. Mol Genet Metab 2016;118:304-9.

11. Lin S-P, Lin H-Y, Wang T-J, Chang C-Y, Lin C-H, Huang S-F, et al. A pilot newborn screening program for mucopolysaccharidosis type I in Taiwan. Orphanet J Rare Dis 2013;8:147.

12. Hopkins PV, Campbell C, Klug T, Rogers S, Raburn-Miller J, Kiesling J. Lysosomal storage disorder screening implementation: findings from the first six months of full population pilot testing in Missouri. J Pediatr 2015;166:172-7.

13. Burton BK, Charrow J, Hoganson GE, Waggoner D, Tinkle B, Braddock SR, et al. Newborn screening for lysosomal storage disorders in Illinois: the initial 15-month experience. J Pediatr 2017;190:130-5.

14. Taylor JL, Clinard K, Powell CM, Rehder C, Young SP, Bali D, et al. The North Carolina experience with mucopolysaccharidosis type I newborn screening. J Pediatr 2019;211:193-200.e2.

15. U.S. Health Resources \& Services Administration. Recommended Uniform Screening Panel. https://www.hrsa.gov/ advisory-committees/heritable-disorders/rusp/index.html. [Accessed March 11, 2020].

16. Burlina AB, Polo G, Salviati L, Duro G, Zizzo C, Dardis A, et al. Newborn screening for lysosomal storage disorders by tandem mass spectrometry in North East Italy. J Inherit Metab Dis 2018;41:209-19.

17. Burlina AB, Polo G, Rubert L, Gueraldi D, Cazzorla C, Duro G, et al. Implementation of Second-tier tests in newborn screening for lysosomal disorders in North Eastern Italy. Int J Neonatal Screen 2019;5:24.

18. Donati MA, Pasquini E, Spada M, Polo G, Burlina A. Newborn screening in mucopolysaccharidoses. Ital J Pediatr 2018;44:126.

19. Zhang H, Young SP, Millington DS. Quantification of glycosaminoglycans in urine by isotope-dilution liquid chromatographyelectrospray ionization tandem mass spectrometry. Curr. Protoc Hum Genet Chapter 2013;17:Unit 17.12.

20. Zhang H, Young SP, Auray-Blais C, Orchard PJ, Tolar J, Millington DS. Analysis of glycosaminoglycans in cerebrospinal fluid from patients with mucopolysaccharidoses by isotope-dilution ultraperformance liquid chromatography-tandem mass spectrometry. Clin Chem 2011;57:1005-12.

21. Tebani A, Zanoutene-Cheriet L, Adjtoutah Z, Abily-Donval L, Brasse-Lagnel C, Laquerrière A, et al. Clinical and molecular characterization of patients with mucopolysaccharidosis type I in an Algerian series. Int J Mol Sci 2016;17:E743.

22. Minter Baerg MM, Stoway SD, Hart J, Mott L, Peck DS, Nett SL, et al. Precision newborn screening for lysosomal disorders. Genet Med 2018;20:847-54.

23. Gelb MH, Lukacs Z, Ranieri E, Schielen PC. Newborn screening for lysosomal storage disorders: methodologies for measurement of enzymatic activities in dried blood spots. Int J Neonatal Screen 2019;5:1.

24. Polo G, Burlina AP, Ranieri E, Colucci F, Rubert L, Pascarella A, et al. Plasma and dried blood spot lysosphingolipids for the diagnosis of different sphingolipidoses: a comparative study. Clin Chem Lab Med 2019;61:1863-74.
25. Turgeon CT, Orsini JJ, Sanders KA, Magera MJ, Langan TJ, Escolar $M L$, et al. Measurement of psychosine in dried blood spots a possible improvement to newborn screening programs for Krabbe disease. J Inherit Metab Dis 2015;38:923-9.

26. Chuang C-K, Lin H-Y, Wang T-J, Huang Y-H, Chan M-J, Liao H-C, et al. Status of newborn screening and follow up investigations for mucopolysaccharidoses I and II in Taiwan. Orphanet J Rare Dis 2018;13:84.

27. McHugh DM, Cameron CA, Abdenur JE, Abdulrahman M, Adair 0 , Al Nuaimi SA, et al. Clinical validation of cutoff target ranges in newborn screening of metabolic disorders by tandem mass spectrometry: a worldwide collaborative project. Genet Med 2011;13:230-54.

28. Taylor JL, Lee S. Lessons learned from newborn screening in pilot studies. N C Med J 2019;80:54-58.

29. Tomatsu S, Montaño AM, Oguma T, Dung VC, Oikawa H, Gutiérrez ML, et al. Validation of disaccharide compositions derived from dermatan sulfate and heparan sulfate in mucopolysaccharidoses and mucolipidoses II and III by tandem mass spectrometry. Mol Genet Metab 2010;99:124-31.

30. de Ruijter J, de Ru MH, Wagemans T, Ijlst L, Lund AM, Orchard PJ, et al. Heparan sulfate and dermatan sulfate derived disaccharides are sensitive markers for newborn screening for mucopolysaccharidoses types I, II and III. Mol Genet Metab 2012;107:705-10.

31. Kubaski F, Suzuki Y, Orii K, Giugliani R, Church HJ, Mason RW, et al. Glycosaminoglycan levels in dried blood spots of patients with mucopolysaccharidoses and mucolipidoses. Mol Genet Metab 2017;120:247-54.

32. Langan TJ, Jalal K, Barczykowski AL, Carter RL, Stapleton M, Orii $\mathrm{K}$, et al. Development of a newborn screening tool for mucopolysaccharidosis type I based on bivariate normal limits: using glycosaminoglycan and alpha-L-iduronidase determinations on dried blood spots to predict symptoms. JIMD Rep 2020;52:35-42.

33. Stapleton M, Kubaski F, Mason RW, Shintaku H, Kobayashi H, Yamaguchi S, et al. Newborn screening for mucopolysaccharidoses: measurement of glycosaminoglycans by LC-MS/MS. Mol Genet Metab Reports 2020;22:100563.

34. Peck L, White P, Studinski F, Ahmad S, Viall S, Siemon H, et al. Incorporation of second-tier biomarker testing improves the specificity of newborn screening for mucopolysaccharidosis type I. Int J Neonatal Screen 2020;6:10.

35. Auray-Blais C, Bhérer P, Gagnon R, Young SP, Zhang HH, An Y, et al. Efficient analysis of urinary glycosaminoglycans by LC-MS/ MS in mucopolysaccharidoses type I, II and VI. Mol Genet Metab 2011;102:49-56.

36. Auray-Blais C, Lavoie P, Tomatsu S, Valayannopoulos V, Mitchell JJ, Raiman J, et al. UPLC-MS/MS detection of disaccharides derived from glycosaminoglycans as biomarkers of mucopolysaccharidoses. Anal Chim Acta 2016;936:139-48.

37. Menkovic I, Marchand A, Boutin M, Auray-Blais C. Neonatal mass urine screening approach for early detection of mucopolysaccharidoses by UPLC-MS/MS. Diagnostics 2019;9:195.

Supplementary Material: The online version of this article offers supplementary material (https://doi.org/10.1515/cclm-2020-0064). 